Document downloaded from:

http://hdl.handle.net/10251/105311

This paper must be cited as:

Martín-García, N.; Vennestrom; Peter Nicolai Ravnborg; Thogersen, JR.; Moliner Marin, M.; Corma Canós, A. (2017). Fe-Containing Zeolites for NH3-SCR of NOx: Effect of Structure, Synthesis Procedure, and Chemical Composition on Catalytic Performance and Stability. Chemistry - A European Journal. 23(54):13404-13414. doi:10.1002/chem.201701742

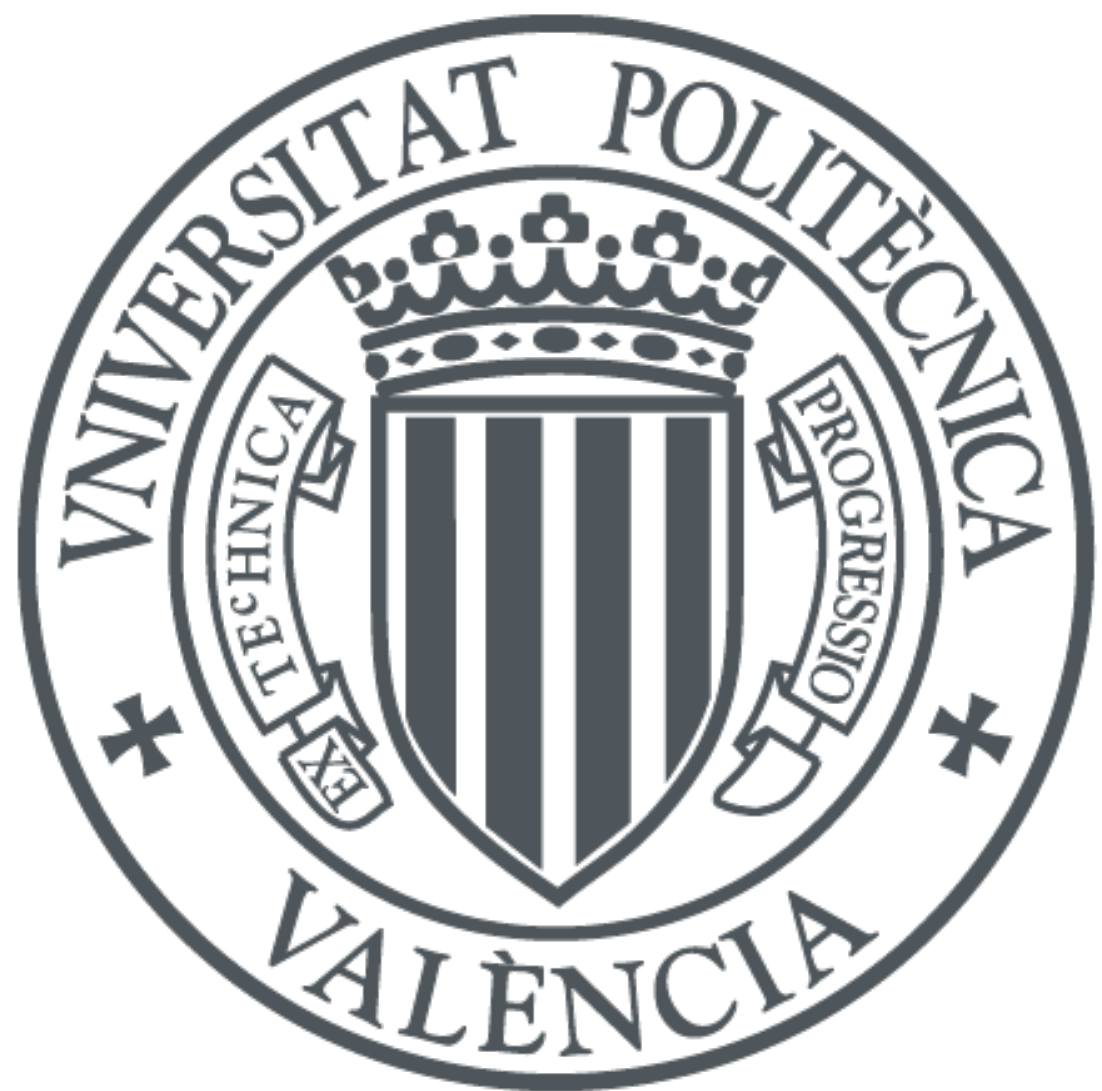

The final publication is available at

http://doi.org/10.1002/chem.201701742

Copyright John Wiley \& Sons

Additional Information 


\title{
Fe-containing zeolites for $\mathrm{NH}_{3}-\mathrm{SCR}$ of $\mathrm{NO}_{x}$ : effect of structure, synthesis procedure and chemical composition on catalytic performance and stability
}

\author{
Nuria Martín, ${ }^{[a]}$ Peter N. R. Vennestrøm, ${ }^{[b]}$ Joakim R. Thøgersen, ${ }^{[b]}$ Manuel Moliner, ${ }^{*[a]}$ Avelino Corma ${ }^{\star}$ \\ [a]
}

\begin{abstract}
The direct preparation of different Fe-containing Beta and $\mathrm{CHA}$ zeolites has been attempted under diverse synthesis conditions, including alkaline and fluoride media, to evaluate the influence of their physico-chemical properties on the selective catalytic reduction (SCR) of $\mathrm{NO}_{x}$ using $\mathrm{NH}_{3}$ as a reductant. From the different Fe-Beta zeolites, the sample prepared in absence of alkali-cations with a Si/Al 13, shows high NO conversion values (>90\%). However, this catalyst suffers from severe deactivation when aged at high temperatures in the presence of steam. The preparation of more hydrophobic Fe-Beta zeolites does not allow improving the catalyst resistance against steaming. In contrast, the Fe-CHA material prepared by one-pot methods under alkaline conditions with a Si/Al 13 using trimethyladamantammonium as template, not only shows excellent catalytic activity but also high hydrothermal stability, especially when sodium cations are selectively removed. Moreover, a Fe-CHA synthesized using the less expensive template tetraethylammonium also results in an active and hydrothermally stable catalyst.
\end{abstract}

\section{Introduction}

Copper and iron-containing zeolites are active catalysts for the selective catalytic reduction of $\mathrm{NO}_{x}$ with ammonia $\left(\mathrm{NH}_{3}-\mathrm{SCR}\right)$ from mobile and stationary sources.$^{[1]}$ In general, the introduction of these metals allows treating exhaust gases at different temperatures. ${ }^{[2]}$ Cu-containing zeolites give better SCR catalytic performance at low-medium temperatures (below $400^{\circ} \mathrm{C}$ ), ${ }^{[3]}$ whereas Fe-containing zeolites provide better SCR performance at high temperatures with improved selectivity (above $400^{\circ} \mathrm{C}$ ). ${ }^{[4]}$ Since high operational temperatures (above $350^{\circ} \mathrm{C}$ ) can be achieved during high diesel-engine loads, but also in the exhaust gases generated in most of the stationary sources, such as power plants or gas turbines, ${ }^{[5]}$ it is interesting to develop efficient and stable Fe-containing zeolite-based $\mathrm{NH}_{3}$-SCR catalysts for hightemperature applications.

The most studied Fe-containing zeolitic SCR catalysts in the last twenty years have been those based on medium-pore ZSM-5 and large-pore Beta zeolites. ${ }^{[1 b, 6]}$ These materials show high SCR catalytic activity, but they present a limited hydrothermal stability when aged at higher temperatures in the presence of steam. ${ }^{[6 \mathrm{e}]}$ The presence of water at high temperatures favors the

\footnotetext{
[a] N. Martin, Dr. M. Moliner, Prof. A. Corma Instituto de Tecnología Química, Universitat Politècnica de València-Consejo Superior de Investigaciones Científicas, Avenida de los Naranjos s/n, 46022 València, Spain E-mail: mmoliner@itq.upv.es, acorma@itq.upv.es

[b] Dr. P. N. R. Vennestrøm, Dr. J. R. Thøgersen Haldor Topsøe A/S, Haldor Topsøes Allé 1, DK-2800 Lyngby, Denmark
}

dealumination of these aluminosilicate zeolites, allowing the progressive clustering of the extra-framework species of iron up to oxidic nanoparticles, resulting in the permanent deactivation of these catalysts. ${ }^{[7]}$ It is worth noting that these Fe-containing ZSM5 or Beta catalysts have been prepared, in general, under postsynthetic ion exchange procedures to introduce the iron species within commercially available zeolites. ${ }^{[8]}$ These commercial zeolites usually present limited Si/Al ratios (between 12-15), relatively small crystal sizes, and large number of structural defects. ${ }^{[8,9]}$ Nevertheless, the ability to synthesize zeolites with different Si/Al molar ratios, crystal sizes or number of structural defects, is very important to control the final properties, since these parameters have direct implications on zeolite polarity and hydrothermal stability. ${ }^{[10]}$

Recently, it has been described that Fe-exchanged small pore chabazite (CHA) zeolite shows not only good SCR catalytic activity, but also remarkably better hydrothermal stability than traditional Fe-exchanged Beta and Fe-ZSM-5 catalysts. ${ }^{[11]}$ The crystalline structure of $\mathrm{CHA}$ zeolite contains large cavities connected through small windows of $3.8 \AA^{.12]}$ The presence of these large cavities could favor the stabilization of the extraframework cationic Fe species, while the presence of the small windows can preclude the metal sintering under ageing conditions, explaining its better resistance against deactivation. Regarding the incorporation of iron species within the $\mathrm{CHA}$ zeolite, ionexchange is, to the best of our knowledge, the only procedure described up to now in the literature, ${ }^{[11]}$ and two-consecutive ionexchange treatments are required to obtain desired exchange levels, under $\mathrm{N}_{2}$ atmosphere to avoid the oxidation of $\mathrm{Fe}^{2+}$ to $\mathrm{Fe}^{3+}$, which could lead to undesired oligomerization of Fe-species. ${ }^{[11]}$ The direct synthesis of iron-containing zeolites eliminates the need for post-synthetic ion-exchange treatments, especially the cumbersome conditions required to obtain iron-loaded small pore zeolites and is therefore desirable. Besides the reduction in the number of preparation steps required to obtain Fe-containing zeolites, the direct preparation could also provide a more homogeneous metal dispersion within the zeolitic crystals compared to traditional ion-exchange procedures. This should be even more important for materials with large crystal sizes and high Si/Al molar ratios, where the metal cations can suffer diffusion limitations or can be poorly charge balanced, respectively. Herein, we study direct synthesis methods of different structured Fe-containing zeolites, particularly Beta and CHA zeolites. Diverse synthesis conditions have been applied to favor the preparation of materials with controlled physico-chemical properties, and their catalytic activity and hydrothermal stability for the $\mathrm{NH}_{3}-\mathrm{SCR}$ of NOx was evaluated. Interestingly, Beta and $\mathrm{CHA}$ zeolites can be prepared within a broad range of Si/Al ratios, crystal sizes, and different amount of structural defects. ${ }^{[13]}$ It could be expected that if these properties can be fine-tuned, their hydrothermal stability and catalytic performance could be 
improved. In this sense, the direct synthesis of the Fe-Beta and Fe-CHA zeolites will be performed following two different synthesis routes, $\mathrm{OH}$ and $\mathrm{F}$ media, under different chemical compositions. In general, it can be stated that the synthesis in $\mathrm{OH}$ media normally gives samples with smaller crystallites and more structural defects, whereas the synthesis in $\mathrm{F}$ media gives samples with less defects and larger crystallites. ${ }^{[13 b, c]}$ Finally, for the synthesis of the Fe-CHA zeolite, both traditional N,N,Ntrimethyladamantammonium (TMAda) as well as the recently discovered commercially available and less expensive tetraethylammonium (TEA) cation, ${ }^{[13 e, f]}$ have been applied as organic structure directing agents (OSDAs).

\section{Results and Discussion}

\section{1.- Study of chemical composition and synthesis procedure on Fe-containing Beta zeolite $\mathrm{NH}_{3}$-SCR performance}

1.1.- Fe-Beta catalysts synthesized in alkaline medium: effect of the Na cations

The standard conditions for the synthesis of Beta zeolite in $\mathrm{OH}$ media include the use of tetraethylammonium (TEA) as OSDA, intermediate Si/Al ratios (i.e. Si/Al 15) and the presence of alkaline cations in the synthesis media (i.e. sodium cations). ${ }^{[13 a, 14]}$ The synthesis of three different Fe-Beta zeolites was therefore carried out in alkaline media using a Si/Al molar ratio of 15 , a Si/Fe molar ratio of 100 , and three different $\mathrm{NaOH} / \mathrm{Si}$ molar ratios in the synthesis gels $(0,0.2,0.4$ for BetaOH_1,_2 and_3, respectively, see Experimental section).

Powder X-ray diffraction (PXRD) patterns show that in all cases Beta zeolite is successfully obtained without the presence of other crystalline impurities (see Figure 1a). Scanning electron microscopy (SEM) images show homogeneous crystals for all the three solids. For BetaOH_1 an average crystal size of 50-100 nm is observed (see Figure 2), whereas BetaOH_2 and BetaOH_3 both show an average crystal size slightly higher $(\sim 200-300 \mathrm{~nm}$, see Figure 2).

Chemical analyses indicate similar Si/Fe molar ratio in the three solids ( 85 , see Table 1 ), which is slightly lower than the theoretical value introduced in the gels (Si/Fe 100) most likely due to the loss of $\mathrm{Si}$ that remain in solution after crystallization. This is consistent with a slight decrease of the Si/Al ratio in the Fe-Beta zeolites when increasing the $\mathrm{NaOH} / \mathrm{Si}$ content (from 13.1 to 9.5 , see Table 1 ), which may be explained by the higher $\mathrm{pHs}$ achieved in the synthesis gels.

The nature and distribution of iron species in the as-prepared and calcined Fe-Beta materials were characterized by diffusereflectance UV-Vis spectroscopy. As it is observed in Figure 3a, the UV-Vis spectra of the as-prepared Fe-Beta samples synthesized in $\mathrm{OH}$ media show the presence of two bands centered at 215 and $240 \mathrm{~nm}$, which have previously been assigned to isolated $\mathrm{Fe}^{3+}$ sites in tetrahedral coordination within the zeolitic framework. ${ }^{[6,15]}$ However, a clear shift of the main band towards higher wavelengths is observed after calcination (see Figure $3 b$ ), indicating the formation of different Fe species. According to the literature, the presence of bands centered at
260-275 nm, has been assigned to isolated $\mathrm{Fe}^{3+}$ species in extraframework positions. ${ }^{[9 b]}$

(A)
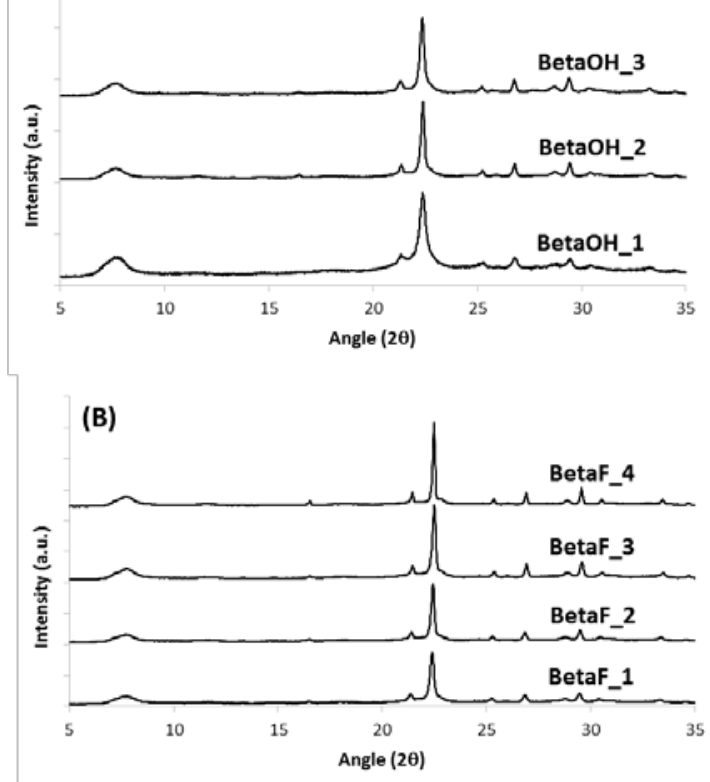

Figure 1. PXRD patterns of the Fe-containing Beta zeolites synthesized in alkaline (A) and fluoride media (B).
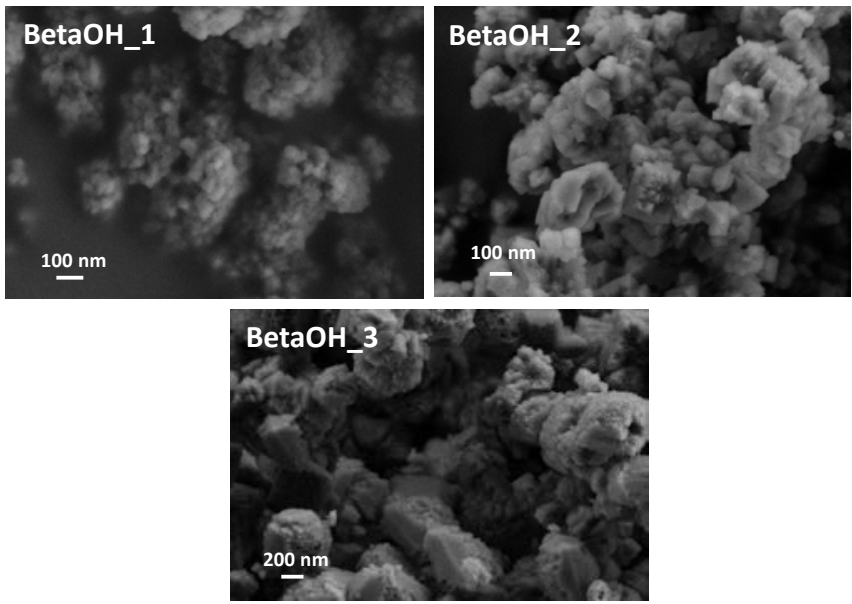

Figure 2. SEM images of the Fe-containing Beta zeolites synthesized in alkaline media.

Since most of the iron species in the calcined Fe-Betas are welldispersed $\mathrm{Fe}^{3+}$ species in extra-framework positions, the catalytic performance in $\mathrm{NH}_{3}$-SCR was evaluated (see Experimental section for reaction conditions). The calcined BetaOH_1 material shows the highest (above 90\%) $\mathrm{NO}_{\mathrm{x}}$ conversion (see BetaOH_1_calc in Figure 4a). In contrast, the other two Fe-Beta catalysts, BetaOH_2 and BetaOH_3, show lower $\mathrm{NO}_{x}$ conversion values. BetaOH_3 shows slightly higher NOx conversion than BetaOH_2, but the highest $\mathrm{NO}_{x}$ conversion values achieved are 
between 60 and $70 \%$ for temperatures above $350^{\circ} \mathrm{C}$ (see Figure 4a).
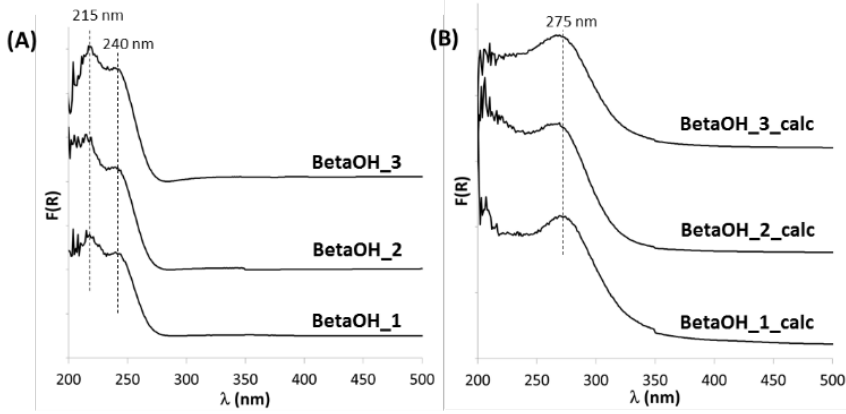

Figure 3. Diffuse-reflectance UV-Vis spectra of the Fe-containing Beta zeolites synthesized in alkaline media in their as-prepared (A) and calcined (B) forms.

In the last years, it has been described for copper containing zeolites that the presence of sodium cations can drastically affect the catalyst stability and activity. ${ }^{[16]}$ Therefore, the absence of extra-framework $\mathrm{Na}$ cations within the BetaOH_1 catalyst could explain the improved catalytic behavior compared to the two sodium-containing Fe-Beta materials (above $1 \% \mathrm{wt}$ of $\mathrm{Na}$, see BetaOH_2 and Beta_OH_3 in Table 1) despite that the deactivation mechanism is different for $\mathrm{Fe}$ - and $\mathrm{Cu}$-containing zeolites. $^{[17]}$

Table 1. Chemical analyses of the Fe-containing Beta zeolites.

\begin{tabular}{ccccccc}
\hline Sample & Si/AI)teor & Si/AI) ICP & Si/Fe)teor & Si/Fe) ICP & $\begin{array}{c}\text { \%wt } \\
\text { Fe }\end{array}$ & $\begin{array}{c}\text { \%wt } \\
\text { Na }\end{array}$ \\
\hline BetaOH_1 & 15 & 13.1 & 100 & 84.7 & 1.02 & 0.02 \\
BetaOH_2 & 15 & 11.9 & 100 & 86.2 & 0.99 & 1.21 \\
BetaOH_3 & 15 & 9.5 & 100 & 87.1 & 0.97 & 1.06 \\
BetaF_1 & 15 & 12.3 & 100 & 72.7 & 1.18 & --- \\
BetaF_2 & 25 & 22.2 & 100 & 82.8 & 1.07 & --- \\
BetaF_3 & 50 & 42.2 & 100 & 77.5 & 1.17 & --- \\
BetaF_4 & INF & INF & 100 & 91.1 & 0.98 & --- \\
\hline
\end{tabular}

The hydrothermal stability of the sodium-free BetaOH_1 sample was studied by subjecting the calcined sample to steam at $600^{\circ} \mathrm{C}$ for 13 hours $\left(10 \% \mathrm{H}_{2} \mathrm{O}, 10 \% \mathrm{O}_{2}\right.$ and $\left.\mathrm{N}_{2}\right)$. The crystalline structure of the BetaOH_1 zeolite remains intact after being steamed at $600^{\circ} \mathrm{C}$ (PXRD pattern not shown), but the catalytic activity of this aged Fe-Beta material shows $\mathrm{NO}_{\times}$conversions values below $50 \%$ under most of the tested reaction temperatures (see white diamonds in Figure 4b), which are remarkably lower than the values achieved by the fresh sodium-free Fe-Beta catalyst $\left(\mathrm{NO}_{x}\right.$ conversions above $90 \%$, see black diamonds in Figure $4 \mathrm{~b}$ ).
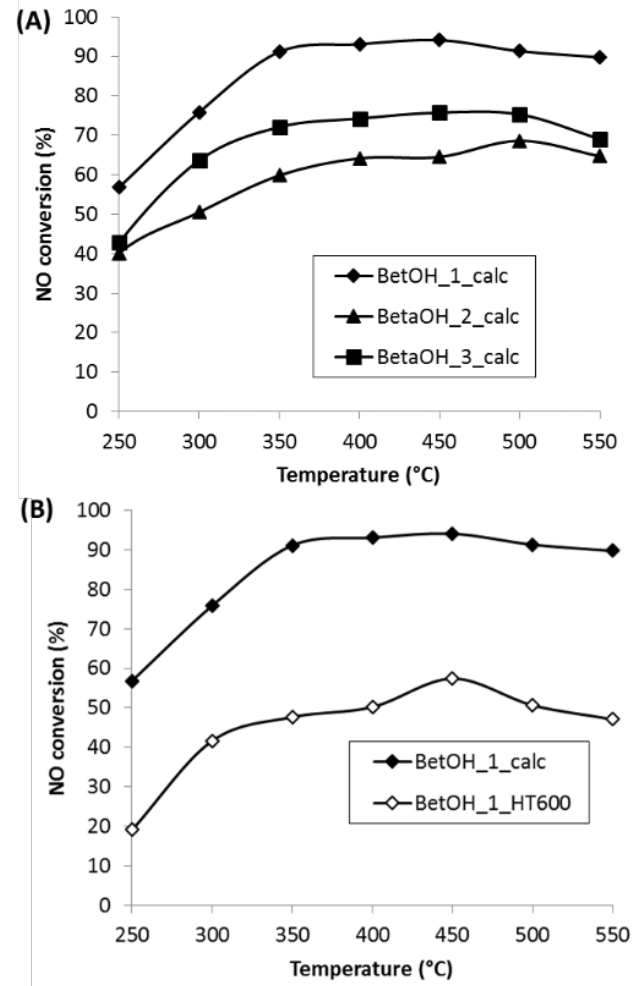

Figure 4. Measured $\mathrm{NO}_{x}$ conversion over the Fe-Beta zeolites synthesized in alkaline conditions $(A)$ and after ageing in presence and absence of water in the reaction feed $(B)$.

If the UV-Vis spectrum of the steamed BetaOH_1 material is analyzed, a broad shoulder between $335-350 \mathrm{~nm}$ is observed, which was not present in the previous calcined material (see Figure S1 in the Supporting Information). Similar bands have been attributed in the literature to the presence of iron in small oligonuclear clusters $\left(\mathrm{Fe}^{3+}{ }_{x} \mathrm{O}_{\mathrm{y}}\right)$. The absence of signals above 400 $\mathrm{nm}$ in the UV-Vis spectrum of the steamed BetaOH_1 sample indicates that large $\mathrm{Fe}_{2} \mathrm{O}_{3}$ particles are not formed after the steaming process. ${ }^{[6,15]}$ These results obtained by UV-Vis spectroscopy indicate that the presence of water during the ageing process favors the agglomeration of the extra-framework isolated Fe species into oligomeric species of Fe, resulting in a catalyst deactivation.

The amount of deactivation for BetaOH_1 is sufficient to follow even at $550^{\circ} \mathrm{C}$, where a decrease in the $\mathrm{NO}_{x}$ conversion is clearly seen within hours (see Figure S2 in Supporting Information).

\section{2.- Fe-Beta catalysts synthesized in fluoride medium: effect of the Si/Al ratio}

Since the deactivation is related to the presence of water, we thought that the more hydrophobic zeolitic crystals of Fe-Beta, with less defects could have an effect on the diffusion of the water molecules through the internal pores of the Beta zeolite and, if so, improve the hydrothermal stability of this type of Fe-containing catalysts. 
It has been broadly described that the preparation of Beta zeolite in fluoride media allows achieving very hydrophobic catalysts with in general, larger crystals and low structural defects. ${ }^{[13 b, c]}$ Having that in mind, the synthesis of four Fe-containing Beta zeolites in fluoride media was carried out, containing a Si/Fe molar ratio of 100 and different Si/Al ratios (15, 25, 50 and INF, see synthesis conditions for BetaF_1, _2, _3, and_4, respectively, in Experimental section).

PXRD reveals that all four samples prepared in fluoride media are highly crystalline Beta zeolites without the presence of impurity phases (see Figure 1b). The crystal size and morphology are seen from the SEM images in Figure 5. As seen, the crystal size clearly depends on the Si/Al ratio introduced in the synthesis gels, achieving an average crystal size of $0.7,1,4$ and $6 \mu \mathrm{m}$, for samples BetaF_1 (Si/Al=15), BetaF_2 (Si/Al=25), BetaF_3 (Si/Al=50) and, BetaF_4 (Si/Al=INF), respectively. Regarding the chemical composition of these Fe-Beta zeolites, ICP analyses indicate that the solids present comparable Si/Al and Si/Fe molar ratios to the theoretical values introduced in the initial synthesis gels (see Table 1).
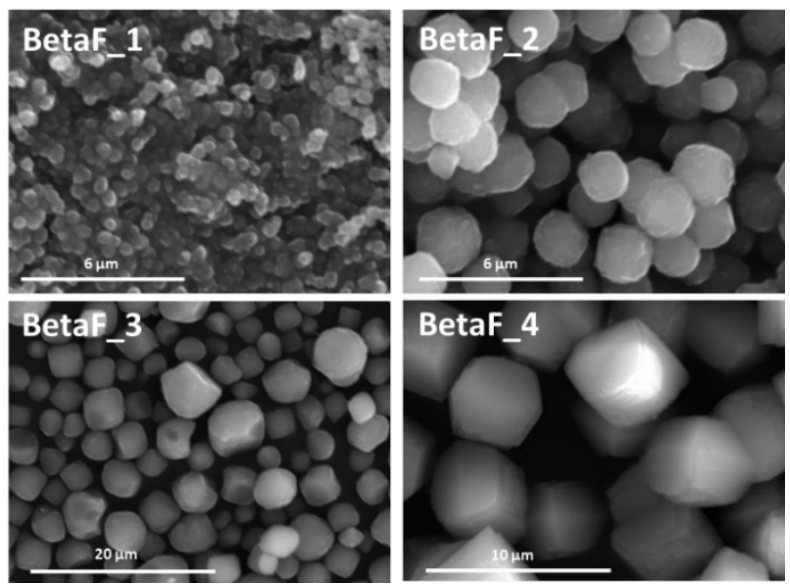

Figure 5. SEM images of the Fe-containing Beta zeolites synthesized in fluoride media.

Similar to the samples prepared in hydroxide media, the asprepared and calcined Fe-Beta materials synthesized in fluoride media were also characterized by diffuse-reflectance UV-Vis spectroscopy to elucidate the nature and distribution of ironspecies. All samples in the as-prepared form show the presence of two main bands centered at 215 and $240 \mathrm{~nm}$ (see Figure 6a), that have been assigned to isolated $\mathrm{Fe}^{3+}$ species in tetrahedral coordination. ${ }^{[6 e, 15]}$ In contrast, some differences are clearly observed in the UV-Vis spectra of the Fe-Beta samples after calcination (see Figure $6 \mathrm{~b}$ ). The samples synthesized with Si/Al ratios of 15 and 25 (see BetaF_1_calc and BetaF_2_calc), show a main band centered at $\sim 270 \mathrm{~nm}$, which has been assigned to isolated $\mathrm{Fe}^{3+}$ species in extra-framework positions, ${ }^{[9 b]}$ whereas the samples synthesized with higher Si/Al ratios, particularly the Al-free Beta (BetaF_4_calc), maintain most of the iron atoms in tetrahedral coordination in the zeolitic framework (see bands at 215 and $240 \mathrm{~nm}$ in Figure 6b). These results indicate that only when iron can be counterbalanced by the presence of framework Al it is dislodged from the tetrahedral framework positions and converted into extra-framework iron species.
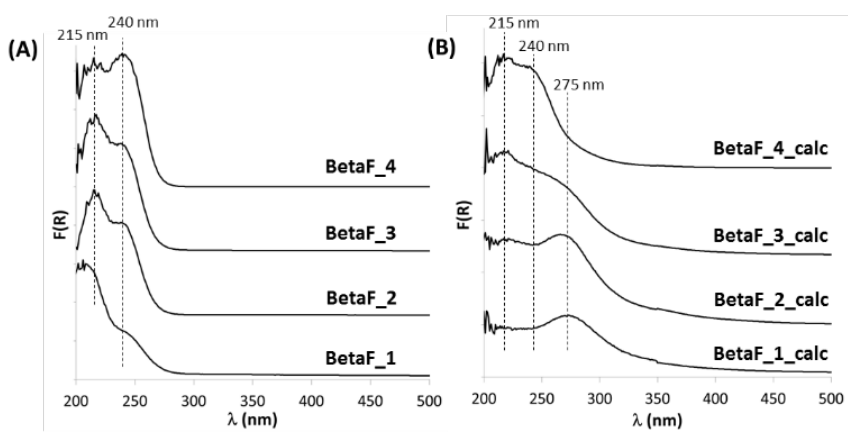

Figure 6. Diffuse-reflectance UV-Vis spectra of the Fe-containing Beta zeolites synthesized in fluoride media in their as-prepared (A) and calcined (B) forms.
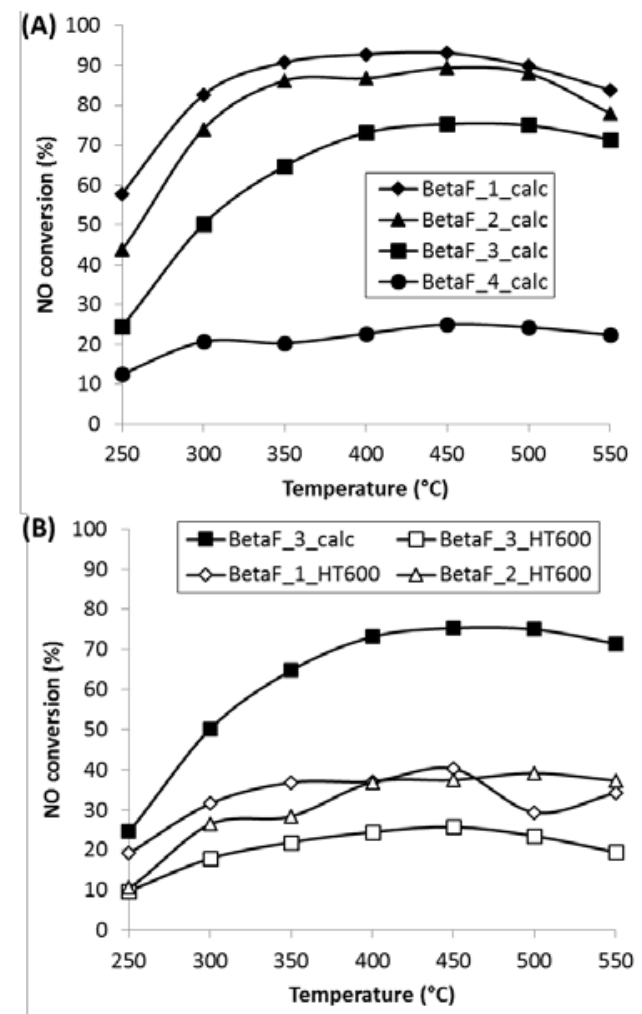

Figure 7. Measured $\mathrm{NO}_{x}$ conversion over the Fe-Beta zeolites synthesized in fluoride media after being calcined in air at $550^{\circ} \mathrm{C}(\mathrm{A})$ and steamed at $600^{\circ} \mathrm{C}$ for $13 \mathrm{~h}(\mathrm{~B})$.

Each of the samples synthesized in fluoride media show different $\mathrm{NO}_{x}$ conversions, as can be seen in Figure 7a. In general, there is a clear relationship between the Si/Al ratio of the Fe-Beta catalysts and their catalytic activity, increasing the NO conversion 
when decreasing the Si/Al ratio (see Figure 7a). The samples containing Si/Al ratios of 15 and 25 (BetaF_1 and BetaF_2, respectively), show $\mathrm{NO}_{x}$ conversion values above $80 \%-90 \%$ at reaction temperatures higher than $300^{\circ} \mathrm{C}$ (see Figure $7 \mathrm{a}$ ). These catalytic results are comparable to those obtained previously for the sodium-free Fe-Beta catalyst synthesized under alkaline conditions (see BetaOH_1 in Figure 4a).

The plausible reason for the higher catalytic activity of the FeBetas with lower Si/Al ratios could be explained by the combination of their lower crystal sizes $(\sim 1 \mu \mathrm{m}$, see Figure 5$)$, and the preferential formation of isolated $\mathrm{Fe}^{3+}$ species in extraframework positions after being calcined (see UV-Vis spectra of BetaF_1_calc and BetaF_2_calc in Figure 6b). Unfortunately, these two very active Fe-Beta catalysts suffer a severe deactivation when steamed at $600^{\circ} \mathrm{C}$ (see BetaF_1_HT600 and BetaF_2_HT600 in Figure 7b). In this sense, their relatively low crystal size, which is comparable to the one achieved previously for the Fe-Beta catalyst synthesized in alkaline medium, could be one of the reasons explaining this substantial catalyst deactivation.

If this is so, the hydrothermal stability of a more hydrophobic FeBeta catalyst must be evaluated to confirm this point. From the different catalysts, the Fe-Beta zeolite synthesized in fluoride media with a Si/Al ratio of 50 , BetaF_3, shows a moderate catalytic activity in its fresh form (NO conversion $\sim 70 \%$, see Figure $7 \mathrm{a})$, but its crystal size is remarkably larger $(\sim 4 \mu \mathrm{m}$, see Figure 5), and then, its hydrophobic nature is also expected to be substantially higher. Indeed, the ${ }^{29}$ Si MAS NMR spectrum of the BetaF_3 sample reveals a much lower content of structural defects [Q3: $(\mathrm{SiO})_{3}-\mathrm{Si}-\mathrm{OH}$, see signal at $-103 \mathrm{ppm}$ in Figure $\mathrm{S} 3$ in Supporting Information], compared to the samples synthesized in alkaline media (see for instance BetaOH_1 in Figure S3 in Supporting Information]). However, an important decrease of the NO conversion also occurs after being aged at $600^{\circ} \mathrm{C}$ for 13 hours (see white squares in Figure 7b).

From the results presented above, introducing various physicochemical properties do not allow the design of steam-stable FeBeta zeolite catalysts for high-temperature $\mathrm{NH}_{3}-\mathrm{SCR}$ applications Iron agglomeration occurs in all cases that leads to severe deactivation. Overall, it has been shown that extra-framework iron species easily diffuses through the open structure of the Beta zeolite, regardless the Si/Al ratio and the hydrophilic/hydrophobic nature of the Beta crystals leading to severe deactivation. Bearing this in mind, it seems natural to constrain the diffusion of the iron species by decreasing the pore size of the zeolite.

\section{2.- Study of chemical composition and synthesis procedure on Fe-containing $\mathrm{CHA}$ zeolite $\mathrm{NH}_{3}$-SCR performance}

In order to decrease the iron-mobility within the zeolite under water-containing $\mathrm{NH}_{3}$-SCR-reaction conditions, it has recently been shown that the use of small-pore cage-based Fe-zeolites as hydrothermally stable catalysts, especially $\mathrm{Fe}-\mathrm{CHA}$ material is beneficial. ${ }^{[11]}$ As stated earlier, the Fe-CHA catalysts reported in the literature have been prepared following a post-synthetic introduction of the cationic species of iron through a two-step solution ion-exchange protocol, ${ }^{[11]}$ which must be performed under $\mathrm{N}_{2}$ protection to avoid the undesired oxidation of $\mathrm{Fe}^{2+}$ to
$\mathrm{Fe}^{3+}$, and the subsequent formation of bulky Fe-complexes. ${ }^{[11]}$ Therefore, the ability to synthesize the Fe-CHA catalyst under direct synthesis methods is interesting, because not only the successive post-synthetic treatments for the incorporation of the iron species would be avoided, but also the metal dispersion within the zeolitic crystals could be improved.
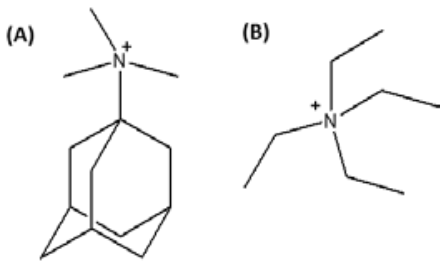

Figure 8. OSDA molecules used for the direct synthesis of the Fe-containing CHA zeolites: N,N,N-trimethyladamantammonium (TMAda, A) and tetraethylammonium (TEA, B).

Table 2. Chemical analyses of the Fe-containing $\mathrm{CHA}$ zeolites.

\begin{tabular}{ccccccc}
\hline Sample & Si/AI)teor & Si/Al)ICP & Si/Fe)teor & Si/Fe)ICP & $\begin{array}{c}\text { \%wt } \\
\text { Fe }\end{array}$ & $\begin{array}{c}\text { \%wt } \\
\text { Na }\end{array}$ \\
\hline ChaOH_1 & 10 & 12.6 & 100 & 92.0 & 0.94 & 1.09 \\
ChaOH_2 & 10 & 8.6 & 100 & 73.8 & 1.13 & 1.88 \\
ChaOH_3 & 15 & 16.4 & 100 & 72.7 & 1.20 & 0.93 \\
ChaOH_4 & 25 & 22.4 & 100 & 66.4 & 1.34 & 0.75 \\
ChaF_1 & 15 & 14.2 & 100 & 91.7 & 0.95 & -- \\
ChaF_2 & 25 & 27.8 & 100 & 107.7 & 0.83 & --- \\
ChaTEA & 10.5 & 7.5 & 100 & 55.1 & 1.70 & 2.40 \\
\hline
\end{tabular}

Interestingly, and similar to the Beta zeolite, CHA can also be synthesized under multiple conditions, including different Si/Al ratios and crystal sizes. ${ }^{[13 e, f, 18]}$ Thus, based on our previous experience, we selected different synthesis conditions to make a direct preparation of Fe-containing $\mathrm{CHA}$ zeolites, both in $\mathrm{OH}$ and $F$ media and to explore the effect of the different synthesis variables on the properties of these SCR catalysts.

\section{1.- Fe-CHA catalysts synthesized in fluoride medium using $N, N, N$-trimethyladamantammonium (TMAdA) as OSDA}

$\mathrm{CHA}$ zeolite in its high-silica and pure silica forms can be synthesized in fluoride media using N,N,Ntrimethyladamantammonium (TMAdA, see Figure 8a) as OSDA. ${ }^{[14 e, f, 18]}$ The iron source was introduced in the synthesis media similar to the direct Fe-Beta synthesis described earlier. The selection of the $\mathrm{Al}$ and Fe amounts were also kept similar to the previous results for Fe-Beta zeolites. Specific synthesis conditions selected for the Fe-CHA materials prepared in fluoride media are described in the Experimental section (see ChaF_1 and ChaF_2 for the CHA materials synthesized with a Si/Al ratio of 15 and 25 , respectively).

As it can be seen in Figure 9b, high-crystalline phase-pure $\mathrm{CHA}$ zeolites are achieved for the syntheses performed in $\mathrm{F}$ media (see the PXRD patterns of ChaF_1 and ChaF_2). The crystal sizes of these $\mathrm{Fe}-\mathrm{CHA}$ materials are relatively large, with average crystal 
sizes of $\sim 7-10 \mu \mathrm{m}$ (see SEM images in Figure 10). Chemical analyses reveal that the final Fe-CHA materials show similar Si/Al and Si/Fe molar ratios to the theoretical values (see ChaF_1 and ChaF_2 in Table 2).

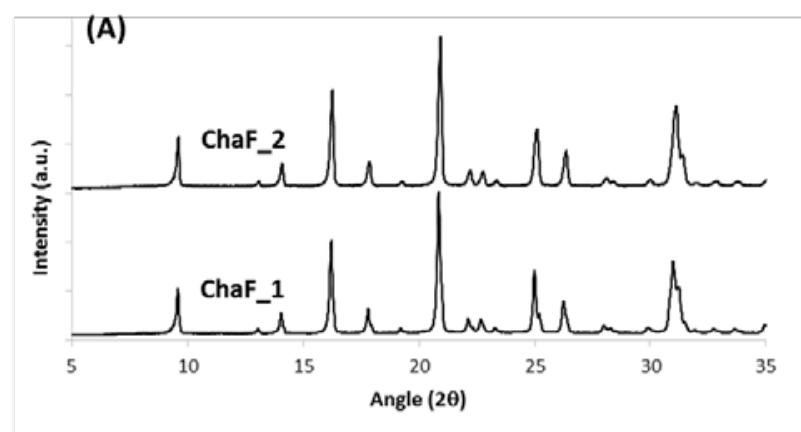

(B)

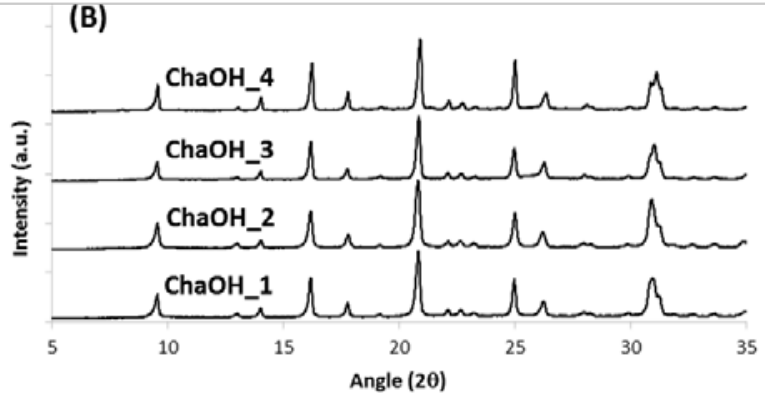

Figure 9. PXRD patterns of the Fe-containing $\mathrm{CHA}$ zeolites synthesized in fluoride $(A)$ and alkaline media $(B)$
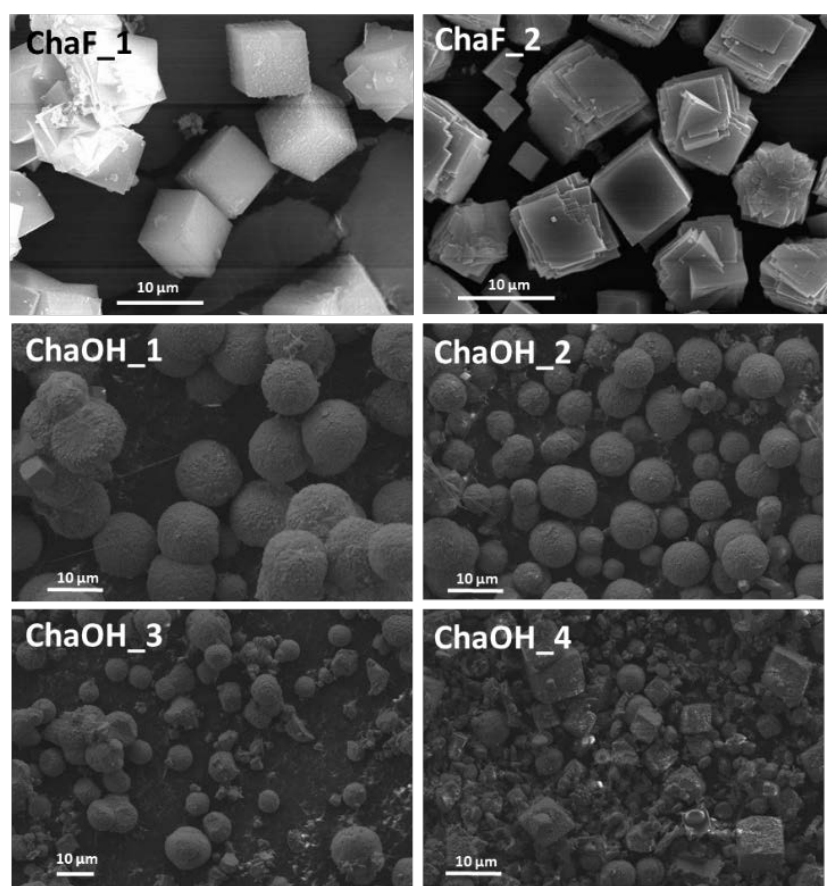

Figure 10. SEM images of the Fe-containing $\mathrm{CHA}$ zeolites synthesized in alkaline media and fluoride media.
The UV-Vis spectra measured on the as-prepared Fe-CHA zeolites show that the iron species are initially in tetrahedral coordination in framework positions (see bands at 215 and 240 $\mathrm{nm}$ in Figure 11a). In comparison, the calcined samples show both iron in tetrahedral framework positions and isolated extraframework iron species seen by the band at $270 \mathrm{~nm}{ }^{[6]}$ (see Figure 11b).
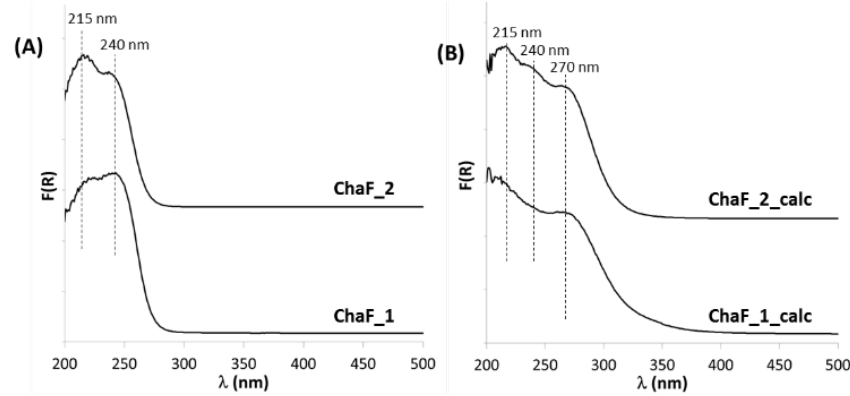

Figure 11. Diffuse-reflectance UV-Vis spectra of the Fe-containing $\mathrm{CHA}$ zeolites synthesized in fluoride media in their as-prepared (A) and calcined (B) forms.

The catalytic performance of these calcined Fe-CHA catalysts was also evaluated in the $\mathrm{NH}_{3}-\mathrm{SCR}$ reaction. Both Fe-CHA materials show medium-low NO conversion values (see Figure S4 in Supporting Information). It would seem that the formation of large crystals with low structural defects favors the stabilization of the $\mathrm{Fe}^{3+}$ species in framework positions even after the calcination treatments, preventing their migration to extra-framework positions that make up the catalytically active sites. This issue was also observed for the Fe-Beta catalysts synthesized in fluoride media with low Al content (see BetaF_3 and BetaF_4 in Figure 6b).

2.2.- Fe-CHA catalysts synthesized in alkaline medium using $N, N, N$-trimethyladamantammonium (TMAdA) as OSDA

At this point, the synthesis of Fe-containing $\mathrm{CHA}$ materials with small crystal sizes and presenting structural defects would provide improved $\mathrm{NH}_{3}-\mathrm{SCR}$ performance. In this sense, the preparation of zeolites in alkaline media in general provides these features.

Based on our previous experience in the synthesis of metalcontaining CHA zeolites, ${ }^{[13]]}$ the following synthesis variables were selected to study the direct crystallization of $\mathrm{Fe}-\mathrm{CHA}$ materials under alkaline conditions: Si/Al $[10,15,25], \mathrm{NaOH} / \mathrm{Si}[0.2,0.4]$ and TMAdAOH/Si $[0.2,0.4]$, maintaining the $\mathrm{Si} / \mathrm{Fe}$ and $\mathrm{H}_{2} \mathrm{O} / \mathrm{Si}$ molar ratios at 100 and 20 , respectively. The crystallization conditions were $160^{\circ} \mathrm{C}$ under static conditions for 10 days (see full synthesis procedure for $\mathrm{ChaOH}_{-} 1,2,2,3$ and 44 in the Experimental section).

The four obtained solids show the characteristic PXRD pattern of the CHA structure (see Figure 9b) without the presence of other crystalline phases. SEM images reveal that the crystal sizes are clearly influenced by the Si/Al ratio. The two samples synthesized with a Si/Al ratio of $10, \mathrm{ChaOH}_{1} 1$ and $\mathrm{ChaOH} 2$, show 
homogeneous spherical particles $(\sim 8 \mu \mathrm{m})$ formed by the aggregation of small crystals of $\sim 0.1 \times 1 \mu \mathrm{m}$ (see $\mathrm{ChaOH} \_1$ and ChaOH_2 in Figure 10). In contrast, the samples synthesized with higher $\mathrm{Si} / \mathrm{Al}$ ratios, especially the sample synthesized with a Si/Al ratio of 25 , show a heterogeneous distribution of crystal sizes (see ChaOH_4 in Figure 10). The chemical composition was measured by ICP, and in general, the Si/Al and Si/Fe molar ratios obtained in the final solids are similar to the initial values introduced in the corresponding synthesis gels (see Table 2).

(A)
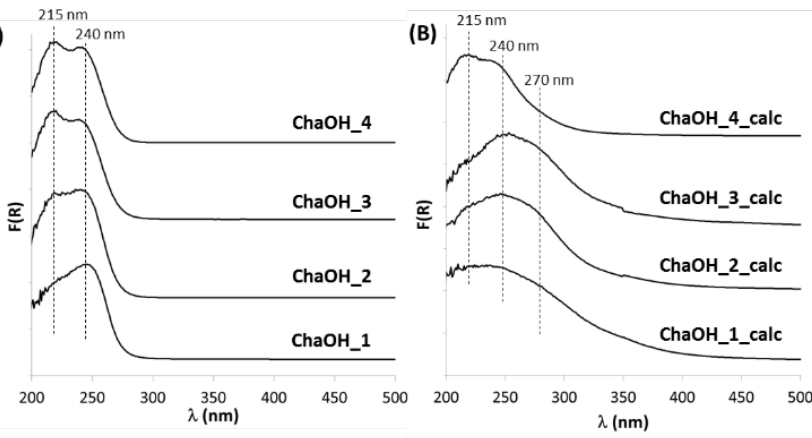

Figure 12. Diffuse-reflectance UV-Vis spectra of the Fe-containing $\mathrm{CHA}$ zeolites synthesized in alkaline media in their as-prepared (A) and calcined (B) forms.

UV-Vis spectra of Fe-CHA samples show a similar trend as was observed for the Fe-Beta materials prepared in $\mathrm{OH}$ media when similar Si/Al ratios are compared. In the as-prepared samples the iron is located in tetrahedral coordination in framework positions (see the bands centered at 215 and $240 \mathrm{~nm}$ in Figure 12a). ${ }^{[15]}$ After calcining at $550^{\circ} \mathrm{C}$ in air, the $\mathrm{Fe}-\mathrm{CHA}$ samples with lower Si/Al ratios ( $10-15$, see $\mathrm{ChaOH}_{-} 1, \ldots 2$ and _3 in Figure $\left.12 \mathrm{~b}\right)$ show a clear displacement of the main bands present in the UVVis spectra toward higher wavelengths ( 250-270 nm), corresponding to isolated iron species in extra-framework positions. $\left.{ }^{[6]}\right]$ In contrast, most of the iron species present in the Fe-CHA sample synthesized with the highest Si/Al ratio ( 25 , see $\mathrm{ChaOH}$ in Figure 12b) remain in framework positions after the calcination process (bands centered at 215 and $240 \mathrm{~nm}$ are still present).

The calcined $\mathrm{ChaOH}$ 1 sample, synthesized with a Si/Al of 10 and the lowest $\mathrm{OH} / \mathrm{Si}$ ratio $(\sim 0.4)$, shows the highest $\mathrm{NO}_{x}$ conversion values (above 90\%) at high reaction temperatures (above $350^{\circ} \mathrm{C}$, see Figure $13 \mathrm{a}$ ). On the other hand, the Fe-CHA samples synthesized with a higher $\mathrm{OH} / \mathrm{Si}$ ratio $(\sim 0.6)$, show different $\mathrm{NO}_{x}$ conversion values depending on the Si/Al ratio, with NO conversion values approaching $80 \%, 70 \%$ and $50 \%$ at reaction temperatures above $350^{\circ} \mathrm{C}$ for the samples synthesized with Si/Al ratios of 10,15 , and 25 , respectively (see Cha_2, _3 and 4 in Figure 13a). Since the iron content is similar in all four Fe-CHA catalysts (Si/Fe 70 , see Table 1 ), their different catalytic behavior is most likely related to the presence of different ironspecies in the final calcined catalysts, as revealed by their UV-Vis spectra (see Figure 12b). In this sense, the more active samples are those containing the main band centered at $270 \mathrm{~nm}$, which have been assigned to extra-framework iron species, while the less active catalyst is that containing the main bands centered at 214-240 nm, which have been assigned to isolated iron species in tetrahedral coordination within the zeolitic framework. Indeed, a very good structure-activity relationship between the normalized area of the UV-Vis spectra centered at $270 \mathrm{~nm}$ and the NO conversion values achieved at $400^{\circ} \mathrm{C}$ is observed not only for the different Fe-CHA zeolites, but also for the above described FeBeta zeolites (see Figure S5 in Supporting Information).
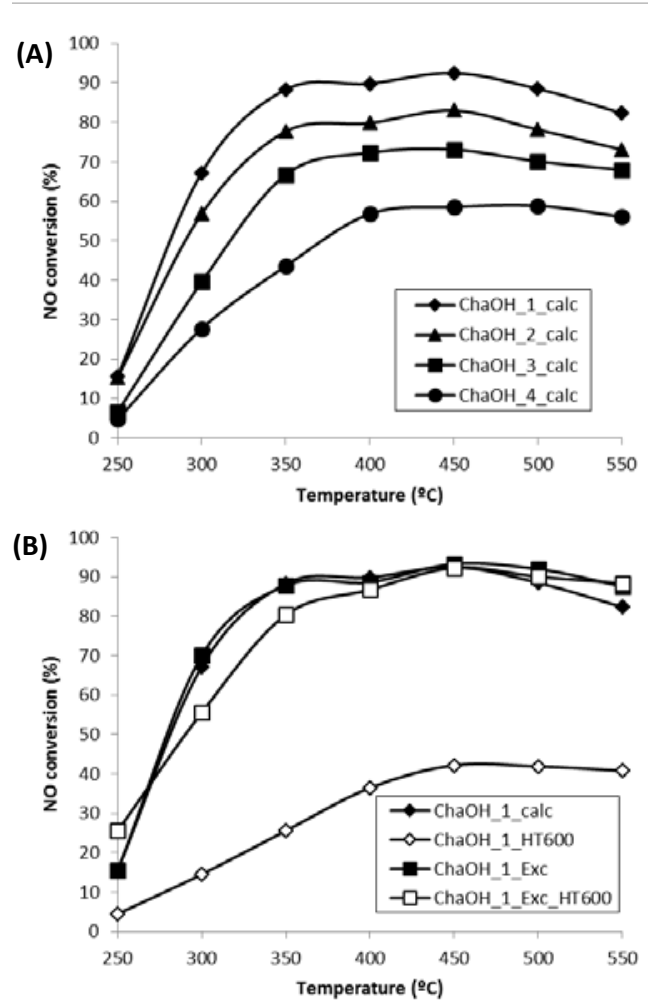

Figure 13. Measured $\mathrm{NO}_{x}$ conversion over the Fe-CHA zeolites synthesized in alkaline media after being calcined in air at $550^{\circ} \mathrm{C}$ (A) and ammoniumexchanged and steamed at $600^{\circ} \mathrm{C}$ for $13 \mathrm{~h}(\mathrm{~B})$

When the most active $\mathrm{Fe}-\mathrm{CHA}$ catalyst, $\mathrm{ChaOH} \_1$, is steamed at $600^{\circ} \mathrm{C}$ for 13 hours, severe deactivation in terms of $\mathrm{NO}_{x}$ conversion is observed (see ChaOH_1_HT600 in Figure 13b). This catalyst contains $\sim 1 \%$ wt of sodium cations (see Table 2 ). In order to see if the competitive charge balance between sodium and iron extra-framework species could be the reason explaining the deactivation of the Fe-CHA catalyst, the calcined $\mathrm{ChaOH}_{-} 1$ catalyst was ion-exchanged with a $1 \mathrm{M}$ aqueous solution of ammonium chloride at $80^{\circ} \mathrm{C}$ for 2 hours, with the aim to selectively remove the sodium cations from the zeolitic sample. SEM-EDX characterization confirms the selective removal of sodium cations while the iron species remain within the $\mathrm{CHA}$ structure. The calcined sodium-free $\mathrm{ChaOH} \_1$ catalyst shows identical high $\mathrm{NO}_{x}$ conversion values in the fresh state as the sodium containing counterpart (see ChaOH_1_Exc in Figure 13b). However, upon 
removal of the sodium ions high $\mathrm{NO}_{x}$ conversion values are maintained after steaming at $600^{\circ} \mathrm{C}$ for $13 \mathrm{~h}$ (see ChaOH_1_Exc_HT600_in Figure 13b). The high $\mathrm{NO}_{x}$ conversion is even maintained after a second steaming cycle, revealing the high hydrothermal stability of the Fe-CHA catalyst synthesized by one-pot methods under alkaline conditions when sodium is removed (see this date in Figure 13b).

\section{3.- Preliminary results of Fe-CHA catalysts synthesized using} inexpensive tetraethylammonium (TEA) cations as OSDA

The preferred methodology (applied above) to synthesize high silica $\mathrm{CHA}$ material requires the use of the expensive $\mathrm{N}, \mathrm{N}, \mathrm{N}$ trimethyladamantammonium (TMAdA, see Figure $8 \mathrm{a}$ ) molecule as OSDA. ${ }^{[13 e, f]} A$ decrease of the manufacturing costs by finding less expensive OSDAs is a matter of interest for industrial applications of CHA-related catalysts. ${ }^{[19]}$ In this sense, we have recently described the synthesis of high-silica $\mathrm{CHA}$ material using the less expensive and more environmentally friendly manufacturing tetraethylammonium (TEA, see Figure $8 b$ ) cation as OSDA, by following a simple zeolite-to-zeolite transformation with a high-silica USY zeolite as the initial silicon and aluminum source. ${ }^{[20]}$

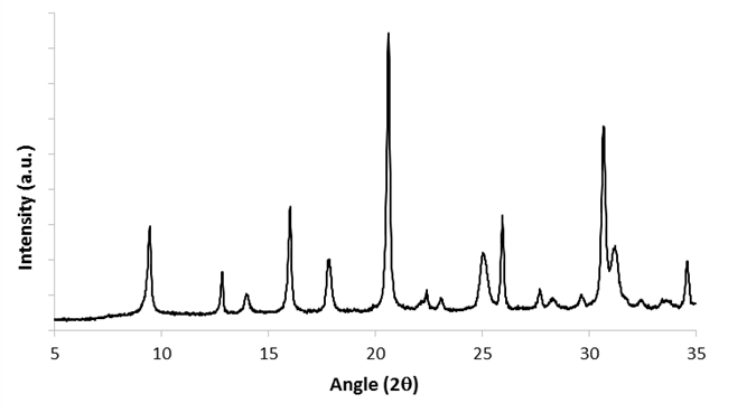

Figure 14. PXRD pattern of the Fe-containing $\mathrm{CHA}$ zeolite synthesized in alkaline medium using TEA as OSDA.

It is natural to extend the recently reported approach with TEA as OSDA to the direct synthesis of the Fe-containing CHA material. To achieve this preparation, the iron source (i.e. iron nitrate) was introduced directly in the synthesis medium, together with TEA and the USY zeolite under the following synthesis conditions: $\mathrm{SiO}_{2} / 0.0047 \mathrm{Al}_{2} \mathrm{O}_{3} / 0.01 \mathrm{Fe} / 0.4 \mathrm{NaOH} / 0.2 \mathrm{TEAOH} / 5 \mathrm{H}_{2} \mathrm{O}$, and the crystallization was conducted at $140^{\circ} \mathrm{C}$ under dynamic conditions for 3 days.

The PXRD pattern of the resultant solid confirms the crystallization of the CHA structure without the presence of other impurity phases (see Figure 14). SEM characterization reveals that the sample consists of large agglomerates of small crystals with a size of 200-300 nm (see Figure S6 in Supporting Information). Similar to all cases described earlier, the asprepared material gives a UV-Vis spectrum indicating the presence of isolated iron species in tetrahedral coordination (bands centered at 215 and $240 \mathrm{~nm}$, see ChaTEA_a.p. in Figure
S7 in Supporting Information). Upon calcination the UV-Vis spectrum shows the preferential formation of extra-framework cationic iron species (band centered at $270 \mathrm{~nm}$, see ChaTEA_calc in Figure S7 in Supporting Information).

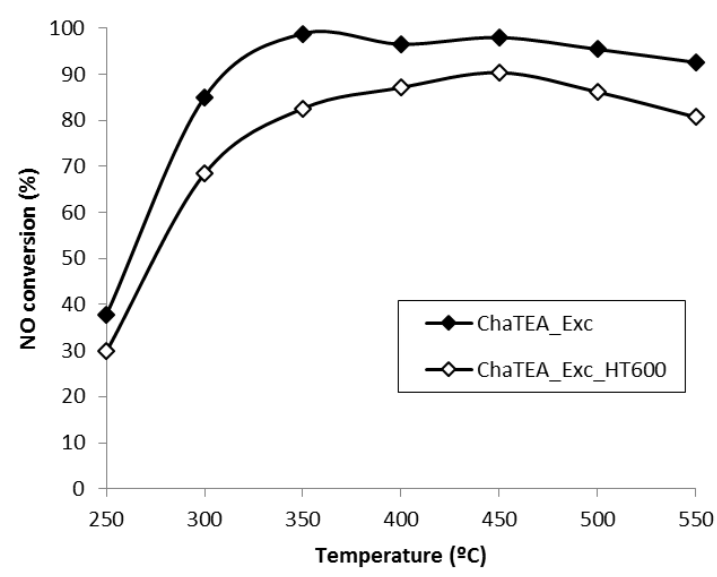

Figure 15. Measured $\mathrm{NO}_{x}$ conversion over the Fe-CHA zeolites synthesized using TEA as OSDA after being calcined in air at $550^{\circ} \mathrm{C}$ and ammoniumexchanged, and steamed at $600^{\circ} \mathrm{C}$ for $13 \mathrm{~h}$.

The Si/Al value is comparable to earlier Fe-Beta and Fe-CHA materials (Si/Al = 7.1). Iron and sodium contents in the final solid are $1.7 \% \mathrm{wt}$ and $2.4 \% \mathrm{wt}$, respectively, which is slightly higher than the prior samples described, but still comparable.

Since the presence of sodium cations within the CHA cavities would affect the hydrothermal stability of the catalyst when subjected to severe conditions, the sodium-containing Fe-CHA catalyst was ion-exchanged with ammonium chloride and then calcined at $500^{\circ} \mathrm{C}$. The complete removal of the sodium cations while maintaining the iron species within the $\mathrm{CHA}$ cavities was verified by EDX-SEM.

The sodium-free Fe-CHA material shows the highest NOx conversion values obtained in this study $\left(\mathrm{NO}_{x}\right.$ conversion values above 95\%) for most of the tested reaction temperatures, especially above $300-350^{\circ} \mathrm{C}$ (see ChaTEA_Exc in Figure 15). Interestingly, this catalyst maintains high catalytic activity with NO conversions approaching $90 \%$ at reaction temperatures above $400^{\circ} \mathrm{C}$ after being steamed at $600^{\circ} \mathrm{C}$ for $13 \mathrm{~h}$ (see ChaTEA_Exc_HT600 in Figure 15). This catalytic behavior is similar to the one observed for the optimum Fe-CHA material synthesized using TMAda cation as OSDA (see $\mathrm{ChaOH}_{-} 1$ in Figure 13b), revealing the high potential of the Fe-containing $\mathrm{CHA}$ material synthesized using TEA as OSDA for the $\mathrm{NH}_{3}-\mathrm{SCR}$ of $\mathrm{NO}_{x}$.

\section{Conclusions}

1) The direct synthesis of the Fe-containing Beta zeolite under alkaline conditions in the absence of sodium cations was successfully achieved by introducing iron in the synthesis gel. The catalytic performance was evaluated showing high conversion 
values (above $90 \%$ ) in the $\mathrm{NH}_{3}-\mathrm{SCR}$ of $\mathrm{NO}_{\mathrm{x}}$. However, this type of catalyst suffers from severe deactivation when aged in presence of steam at high temperatures supposedly due to agglomeration of iron species. In addition, a continuous time-onstream (TOS) catalyst deactivation can also be observed for the fresh Fe-Beta catalyst due to the effect of water under reaction conditions during extended TOS at a given temperature.

2) More hydrophobic Fe-Beta zeolites were prepared in fluoride media with similar $\mathrm{NO}_{x}$ catalytic activities in their fresh form, but they also suffer from severe catalyst deactivation after being aged with steam at high temperatures.

3) The direct synthesis of the Fe-containing CHA materials was also successfully achieved using different synthesis conditions, including fluoride and alkaline media. The Fe-CHA materials synthesized in fluoride media show large crystal sizes $(\sim 10 \mu \mathrm{m})$ and most of the iron species remain in tetrahedral coordination after the thermal treatments, resulting in catalysts with low $\mathrm{NO}_{x}$ conversion values when tested in the $\mathrm{NH}_{3}-\mathrm{SCR}$ reaction. Ironcontaining $\mathrm{CHA}$ materials synthesized following direct methodologies under sodium-containing alkaline conditions show good catalytic activities, especially those presenting Si/Al ratios close to 10 . The hydrothermal stability of these materials is remarkably improved by removing the sodium cations.

4) Finally, the direct synthesis approach of Fe-CHA was also successfully adapted using the less expensive and commercially available TEA molecule as OSDA. After removal of the sodium ions, this material provides excellent $\mathrm{NO}_{x}$ conversion values, and further showed high hydrothermal stability.

\section{Experimental Section}

\section{Zeolite synthesis \\ - Fe-containing Beta zeolite in alkaline media}

The required amount of a $35 \% w t$ aqueous solution of tetraethylammonium hydroxide (TEAOH, Sigma-Aldrich) and a 50\%wt aqueous solution of tetraethylammonium bromide (TEABr, Sigma-Aldrich) were mixed. Then, the required content of a colloidal suspension of silica in water (40\%wt, LUDOX-AS, Sigma-Aldrich) and alumina (75\%wt, Condea) were added, and the resultant mixture maintained under stirring for 15 minutes. Finally, the required amount of a $5 \%$ wt aqueous solution of iron (III) nitrate $\left[\mathrm{Fe}\left(\mathrm{NO}_{3}\right)_{3}\right.$, Sigma Aldrich, 98\%] was added, and the synthesis mixture was maintained under stirring the required time to evaporate the excess of water until achieving the desired gel concentration. The final gel compositions were:

BetaOH_1: [Si : 0.067 Al: $0.01 \mathrm{Fe}: 0.2 \mathrm{TEAOH}: 0.2 \mathrm{TEABr}: 20 \mathrm{H}_{2} \mathrm{O}: 0$ $\mathrm{NaOH}]$

BetaOH_2: [Si : 0.067 Al: $0.01 \mathrm{Fe}: 0.2 \mathrm{TEAOH}: 0.2 \mathrm{TEABr}: 20 \mathrm{H}_{2} \mathrm{O}: 0.2$ $\mathrm{NaOH}]$

BetaOH_3: [Si : 0.067 Al: $0.01 \mathrm{Fe}: 0.2 \mathrm{TEAOH}: 0.2 \mathrm{TEABr}: 20 \mathrm{H}_{2} \mathrm{O}: 0.4$ $\mathrm{NaOH}]$

The resultant gels were charged into stainless steel autoclaves with a Teflon liner. The crystallizations were then conducted at $140^{\circ} \mathrm{C}$ for 7 days under static conditions. The solid product was filtered, washed with abundant water, and dried and finally calcined in air at $550^{\circ} \mathrm{C}$ for $4 \mathrm{~h}$.
- Fe-containing Beta zeolite in fluoride media

The required content of a colloidal suspension of silica in water (40\%wt, LUDOX-AS, Sigma-Aldrich) and alumina (75\%wt, Condea) were mixed with a $35 \%$ wt aqueous solution of tetraethylammonium hydroxide (TEAOH, Sigma-Aldrich), and the resultant mixture maintained under stirring for 15 minutes. Then, the required amount of a $5 \%$ wt aqueous solution of iron (III) nitrate $\left[\mathrm{Fe}\left(\mathrm{NO}_{3}\right)_{3}\right.$, Sigma Aldrich, 98\%] was added, and the synthesis mixture was maintained under stirring for additional 30 minutes. Finally, the required content of a $10 \%$ wt aqueous solution of ammonium fluoride $\left(\mathrm{NH}_{4} \mathrm{~F}\right.$, Sigma-Aldrich) was added, and the mixture maintained under stirring the required time to evaporate the excess of water until achieving the desired gel concentration. The final gel compositions were:

\section{BetaF_1: [Si : $0.067 \mathrm{Al}: 0.01 \mathrm{Fe}: 0.50 \mathrm{TEAOH}: 0.50 \mathrm{NH}_{4} \mathrm{~F}: 7 \mathrm{H}_{2} \mathrm{O}$ ]}

BetaF_2: [Si : $0.033 \mathrm{Al}: 0.01 \mathrm{Fe}: 0.50 \mathrm{TEAOH}: 0.50 \mathrm{NH}_{4} \mathrm{~F}: 7 \mathrm{H}_{2} \mathrm{O}$ ]

BetaF_3: [Si : $0.020 \mathrm{Al}: 0.01 \mathrm{Fe}: 0.50 \mathrm{TEAOH}: 0.50 \mathrm{NH}_{4} \mathrm{~F}: 7 \mathrm{H}_{2} \mathrm{O}$ ]

BetaF_4: [Si : $0.000 \mathrm{Al}: 0.01 \mathrm{Fe}: 0.50 \mathrm{TEAOH}: 0.50 \mathrm{NH}_{4} \mathrm{~F}: 7 \mathrm{H}_{2} \mathrm{O}$ ]

The resultant gels were charged into stainless steel autoclaves with a Teflon liner. The crystallizations were then conducted at $140^{\circ} \mathrm{C}$ for 10 days under static conditions. The solid product was filtered, washed with abundant water, and dried and finally calcined in air at $550^{\circ} \mathrm{C}$ for $4 \mathrm{~h}$.

- Fe-containing CHA zeolite in alkaline media using $N, N, N$ trimethyladamantammonium (TMAda)

The synthesis of the N,N,N-trimethyladamantammonium (TMAda) has been performed according to previous reports in the literature. ${ }^{[13 e, f]}$

The required amount of a $17 \%$ wt aqueous solution of $\mathrm{N}, \mathrm{N}, \mathrm{N}$ trimethyladamantammonium hydroxide (TMAda) and a $20 \%$ wt aqueous solution of sodium hydroxide ( $\mathrm{NaOH}$, Sigma-Aldrich) were mixed. Then, the required content of a colloidal suspension of silica in water (40\%wt, LUDOX-AS, Sigma-Aldrich) and alumina (75\%wt, Condea) were added, and the resultant mixture maintained under stirring for 15 minutes. Finally, the required amount of a $5 \%$ wt aqueous solution of iron (III) nitrate [Fe( $\left(\mathrm{NO}_{3}\right)_{3}$, Sigma Aldrich, 98\%] was added, and the synthesis mixture was maintained under stirring the required time to evaporate the excess of water until achieving the desired gel concentration. The final gel compositions were:

ChaOH_1: [Si : $\left.0.100 \mathrm{Al}: 0.01 \mathrm{Fe}: 0.2 \mathrm{TMAdaOH}: 0.2 \mathrm{NaOH}: 20 \mathrm{H}_{2} \mathrm{O}\right]$

ChaOH_2: [Si : $0.100 \mathrm{Al}: 0.01 \mathrm{Fe}: 0.4 \mathrm{TMAdaOH}: 0.2 \mathrm{NaOH}: 20 \mathrm{H}_{2} \mathrm{O}$ ]

ChaOH_3: [Si : 0.067 Al: $0.01 \mathrm{Fe}: 0.4 \mathrm{TMAdaOH}: 0.2 \mathrm{NaOH}: 20 \mathrm{H}_{2} \mathrm{O}$ ]

ChaOH_4: [Si : $\left.0.040 \mathrm{Al}: 0.01 \mathrm{Fe}: 0.4 \mathrm{TMAdaOH}: 0.4 \mathrm{NaOH}: 20 \mathrm{H}_{2} \mathrm{O}\right]$

The resultant gels were charged into stainless steel autoclaves with a Teflon liner. The crystallizations were then conducted at $160^{\circ} \mathrm{C}$ for 10 days under static conditions. The solid products were filtered, washed with abundant water, and dried at $100^{\circ} \mathrm{C}$. The as-prepared solids were calcined in air at $550^{\circ} \mathrm{C}$ for $4 \mathrm{~h}$

The sodium-containing Fe-CHA materials were mixed with a $1 \mathrm{M}$ aqueous solution of ammonium chloride ( $100 \mathrm{mg}$ solid/1 ml liquid), and the mixture was maintained under stirring at $80^{\circ} \mathrm{C}$ for $5 \mathrm{~h}$. The solid product was filtered, washed with abundant water, and dried at $100^{\circ} \mathrm{C}$. Finally, the solid was calcined in air at $500^{\circ} \mathrm{C}$ for $4 \mathrm{~h}$.

- Fe-containing CHA zeolite in fluoride media 
The synthesis of the N,N,N-trimethyladamantammonium (TMAda) has been performed according to previous reports in the literature. ${ }^{[13 e, f]}$

The required content of a colloidal suspension of silica in water (40\%wt, LUDOX-AS, Sigma-Aldrich) and alumina (75\%wt, Condea) were mixed with a $17 \%$ wt of $\mathrm{N}, \mathrm{N}, \mathrm{N}$-trimethyladamantammonium hydroxide (TMAda), and the resultant mixture maintained under stirring for 15 minutes. Then, the required amount of a $5 \%$ wt aqueous solution of iron (III) nitrate [Fe( $\left(\mathrm{NO}_{3}\right)_{3}$, Sigma Aldrich, 98\%] was added, and the synthesis mixture was maintained under stirring for additional 30 minutes. Finally, the required content of a $10 \%$ wt aqueous solution of ammonium fluoride $\left(\mathrm{NH}_{4} \mathrm{~F}\right.$, SigmaAldrich) was added, and the mixture maintained under stirring the required time to evaporate the excess of water until achieving the desired gel concentration. The final gel compositions were:

ChaF_1: [Si : $0.067 \mathrm{Al}: 0.01 \mathrm{Fe}: 0.50 \mathrm{TMAdaOH}: 0.50 \mathrm{NH}_{4} \mathrm{~F}: 5 \mathrm{H}_{2} \mathrm{O}$ ]

ChaF_2: [Si : $0.04 \mathrm{Al}: 0.01 \mathrm{Fe}: 0.50 \mathrm{TMAdaOH}: 0.50 \mathrm{NH}_{4} \mathrm{~F}: 5 \mathrm{H}_{2} \mathrm{O}$ ]

The resultant gels were charged into stainless steel autoclaves with a Teflon liner. The crystallizations were then conducted at $150^{\circ} \mathrm{C}$ for 14 days under static conditions. The solid products were filtered, washed with abundant water, and dried at $100^{\circ} \mathrm{C}$. The as-prepared solids were calcined in air at $550^{\circ} \mathrm{C}$ for $4 \mathrm{~h}$

- Fe-containing CHA zeolite in alkaline media using tetraethylammonium (TEA)

$0.394 \mathrm{~g}$ of a $35 \% \mathrm{wt}$ aqueous solution of tetraethylammonium hydroxide was mixed with $0.375 \mathrm{~g}$ of a $20 \% \mathrm{wt}$ aqueous solution of sodium hydroxide ( $\mathrm{NaOH}$ granulated, Scharlab). The mixture was maintained under stirring 10 minutes for homogenization. Afterwards, $0.300 \mathrm{~g}$ of FAU zeolite $\left(\mathrm{SiO}_{2} / \mathrm{Al}_{2} \mathrm{O}_{3}=21\right)^{[20]}$ was added in the synthesis mixture. Finally, $0.095 \mathrm{~g}$ of a $20 \%$ wt aqueous solution of iron (III) nitrate [Fe( $\left.\mathrm{NO}_{3}\right)_{3}$, Sigma Aldrich, $98 \%$ ] was added, and the synthesis mixture was maintained under stirring the required time to evaporate the excess of water until achieving the desired gel concentration. The final gel composition was $\mathrm{SiO}_{2}: 0.047$ $\mathrm{Al}_{2} \mathrm{O}_{3}: 0.4 \mathrm{TEAOH}: 0.2 \mathrm{NaOH}: 5 \mathrm{H}_{2} \mathrm{O}$. The resultant gel was charged into a stainless steel autoclave with a Teflon liner. The crystallization was then conducted at $160^{\circ} \mathrm{C}$ for 7 days under static conditions. The solid product was filtered, washed with abundant amounts of water, dried at $100^{\circ} \mathrm{C}$ and, finally, calcined in air at $550^{\circ} \mathrm{C}$ for $4 \mathrm{~h}$.

The sodium-containing Fe-CHA material was mixed with a $1 \mathrm{M}$ aqueous solution of ammonium chloride (100 mg solid/1 ml liquid), and the mixture was maintained under stirring at $80^{\circ} \mathrm{C}$ for $5 \mathrm{~h}$. The solid product was filtered, washed with abundant water, and dried at $100^{\circ} \mathrm{C}$. Finally, the solid was calcined in air at $500^{\circ} \mathrm{C}$ for $4 \mathrm{~h}$.

\section{Characterization}

The crystallinity of the samples was measured by powder X-ray diffraction (PXRD) with a Panalytical CUBIX diffractometer using monochromatic CuKa $_{1,2}$ radiation $(\lambda=1.5406,1.5444 \AA$; Ka2 / Ka1 intensity ratio=0.5).

The morphology and particle size of the zeolites were characterized by Scanning Electron Microscope (SEM, JEOL JSM-6300).

Chemical composition was determined by inductively coupled plasma atomic absorption spectroscopy (ICP-OES) using a Varian 715-ES

UV-Vis spectra were obtained with a Perkin-Elmer (Lambda 19) spectrometer equipped with an integrating sphere with BaSO4 as reference.
The iron-containing catalysts were treated in a gas mixture containing $10 \%$ $\mathrm{H}_{2} \mathrm{O}, 10 \% \mathrm{O}_{2}$ and $\mathrm{N}_{2}$ for 13 hours at $600^{\circ} \mathrm{C}$

\section{Catalytic test}

The catalytic activity was evaluated for the catalytic reduction of $\mathrm{NO}_{x}$ using $\mathrm{NH}_{3}$ in a fixed bed, quartz tubular reactor of $1.2 \mathrm{~cm}$ of diameter and $20 \mathrm{~cm}$ of length. The catalysts were tested using $40 \mathrm{mg}$ with a sieve fraction of $0.25-0.42 \mathrm{~mm}$. The catalysts were introduced in the reactor, heated up to $550^{\circ} \mathrm{C}$ in a $300 \mathrm{ml} / \mathrm{min}$ flow of nitrogen and maintained at this temperature for one hour. Afterwards, 50 ppm NO, 60 ppm $\mathrm{NH}_{3}, 10 \% \mathrm{O}_{2}$ and $10 \% \mathrm{H}_{2} \mathrm{O}$ was admitted over the catalyst while maintaining a flow of $300 \mathrm{ml} / \mathrm{min}$. The temperature was then decreased stepwise between 550 and $250^{\circ} \mathrm{C}$. The conversion of NO was measured under steady state conversion at each temperature using a chemiluminiscence detector (Thermo 62C).

\section{Acknowledgements}

This work has been supported by Haldor Topsøe A/S, by the Spanish Government-MINECO through "Severo Ochoa" (SEV 2012-0267), and MAT2015-71261-R, by the European Union through ERC-AdG-2014-671093 (SynCatMatch); and by the Fundación Ramón Areces through a research contract of the "Life and Materials Science" program. N. M. thanks MINECO for economical support through pre-doctoral fellowship (BES-2013064347). The authors thank Isabel Millet for technical support.

Keywords: Iron-zeolites $\bullet$ Beta $\bullet$ Chabazite $\bullet$ selective catalytic reduction $\bullet$ heterogeneous catalysis

[1] a) S. Brandenberger, O. Kröcher, A. Tissler, R. Althoff, Catal. Rev. Sci. Eng., 2008, 50, 492; b) M. Iwamoto, H. Furukawa, Y. Mine, F. Uemura S.I. Mikuriya, S. Kagawa, J. Chem. Soc., Chem. Commun., 1986, 1272.

[2] A. M. Beale, F. Gao, I. Lezcano-Gonzalez, C. H. F. Peden, J. Szanyi, Chem. Soc. Rev., 2015, 44, 7371.

[3] a) D. W. Fickel, E. D'Addio, J. A. Lauterbach, R. F. Lobo, Appl. Catal. B 2011, 102, 441; b) M. Moliner, C. Martínez, A. Corma, Chem. Mater. 2014, 26, 246; c) J. H. Kwak, R. G. Tonkyn, D. H. Kim, J. Szanyi, C. H. F. Peden, J. Catal., 2010, 275, 187.

[4] F. Gao, Y. Zheng, R. K. Kukkadapu, Y. Wang, E. D. Walter, B. Schwenzer, J. Szanyi, C. H. F. Peden, ACS Catal., 2016, 6, 2939.

[5] a) M. Colombo, I. Nova, E. Tronconi, Catal. Today, 2010, 151, 223; b) P. S. Metkar, M. P. Harold, V. Balakotaiah, Appl. Catal. B, 2012, 111-112, 67.

[6] a) R. Q. Long, R. T. Yang, J. Am. Chem. Soc., 1999, 121, 5595; b) R. Q. Long, R. T. Yang, Catal. Letters, 2001, 74, 201; c) A. Ma, W. Grünert, Chem. Commun., 1999, 71; d) R. Nedyalkova, S. Shawn, M. Skoglundh L. Olsson, Appl. Catal. B, 2013, 138-139, 373; e) J. Perez-Ramirez, J. C. Groen, A. Bruckner, M. S. Kumar, U. Bentrup, M. N. Debbagh, L. A Villaescusa, J. Catal., 2005, 232, 318.

[7] a) J. Perez-Ramirez, G. Mul, F. Kapteijn, J. A. Moulijn, A. R. Overweg, A Domenech, A. Ribera, I. W. C. E. Arends, J. Catal, 2002, 207, 113; b) G Berlier, G. Spoto, S. Bordiga, G. Ricchiardi, P. Fisicaro, A. Zecchina, I. Rossetti, E. Selli, L. Forni, E. Giamello, C. Lamberti, J. Catal., 2002, 208, 64.

[8] a) G. Delahay, M. Mauvezin, B. Coq, S. Kieger, J. Catal., 2001, 202, 156 b) M. Mauvezin, G. delahay, B. Coq, S. Kieger, J. C. Jumas, J. OlivierFourcade, J. Phys. Chem. B., 2001, 105, 928; c) G. Mul, M. W. Zandbergen, F. Kapteijn, J. A. Moulijn, J. Perez-Ramirez, Catal. Lett., 
2004, 93, 113; d) P. Marturano, L. Drozdova, A. Kogelbauer, R. Prins, J. Catal., 2000, 190, 460

[9] a) El-M. El-Malki, R. A van Santen, W. M. H Sachtler, J. Catal., 2000 196, 212; b) M. S. Kumar, M. Schwidder, M. Grünert, A. Brückner, J. Catal., 2004, 227, 384; c) R. Martinez-Franco, C. Paris, M. E. MartinezArmero, C. Martinez, M. Moliner, A. Corma, Chem. Sci., 2016, 7, 102.

[10] a) J. Stelzer, M. Paulus, M. Hunger, J. Weitkamp, Micropor. Mesopor Mater., 1998, 22, 1; b) T. Blasco, M. A. Camblor, A. Corma, P. Esteve, J. M. Guil, A. Martínez, J. A. Perdigón-Melón, S. Valencia, J. Phys. Chem. B, 1998, 102, 75; c) A. Corma, M. E. Domine, S. Valencia, J. Catal., 2003, 215, 294; d) M. Moliner, Y. Roman-Leshkov, M. E. Davis, Proc. Natl. Acad. Sci. USA, 2010, 107, 6164.

[11] a) F. Gao, M. Kollar, R. K. Kukkadapu, N. M. Washton, Y. Wang, J. Szanyi, C. H. F. Peden, Appl. Catal. B, 2015, 164, 407; b) F. Gao, Y. Zheng, R. K. Kukkadapu, Y. Wang, E. D: Walter, B. Scwenzer, J. Szanyi, C. H. F. Peden, ACS Catal., 2016, 6, 2939.

[12] J. V. Smith, Acta Cryst., 1962, 15, 835.

[13] a) M. M. J. Treacy, J. M. Newsam, Nature, 1988, 332, 249; b) M. A Camblor, A. Corma, S. Valencia, Chem. Commun., 1996, 2365; c) M. A.
Camblor, A. Corma, S. Valencia, J. Mater. Chem., 1998, 8, 2137; d) S. I. Zones, J. Chem. Soc. Faraday Trans., 1991, 87, 3709; e) S. I. Zones U.S. Pat., 4544538, 1985; f) R. Martinez-Franco, M. Moliner, J. R. Thogersen, A. Corma, ChemCatChem., 2013, 5, 3316.

[14] M. Moliner, J.M. Serra, A. Corma, E. Argente, S. Valero, V. Botti, Micropor. Mesopor. Mater., 2005, 78, 73.

[15] S. Bordiga, R. Buzonni, F. Geobaldo, C. Lamberti, E. Giamello, A Zecchina, G. Leofanti, G. Petrini, G. Tozzola, G. Vlaic, J. Catal., 1996 $158,486$.

[16] L. Xie, F. Liu, X. Shi, F. S. Xiao, H. He, Appl. Catal. B, 2015,179, 206

[17] P. N. R. Vennestrøm, T. V. W. Janssens, A. Kustov, M. Grill, A. Puig Molina, L. F. Lundegaard, R. R. Tiruvalam, P. Concepción, A. Corma, J. Catal., 2014, 309, 477

[18] a) M. J. Diaz-Cabañas. P. A. Barret, M. A. Camblor, Chem. Commun., 1998, 1881; b) E. A. Eilertsen, B. Arstad, S. Svelle, K. P. Lillerud, Micropor. Mesopor. Mater., 2012, 153, 94

[19] S. I. Zones, Micropor. Mesopor. Mater., 2011, 144, 1.

[20] N. Martin, M. Moliner, A. Corma, Chem. Commun., 2015, 51, 9965. 


\section{Entry for the Table of Contents}

\section{FULL PAPER}

The direct preparation of different Fecontaining zeolites, Beta and $\mathrm{CHA}$, has been attempted under diverse synthesis conditions, including alkaline and fluoride media, to evaluate the influence of their physicochemical properties on the selective catalytic reduction $\mathrm{NO}_{x}$ using $\mathrm{NH}_{3}$

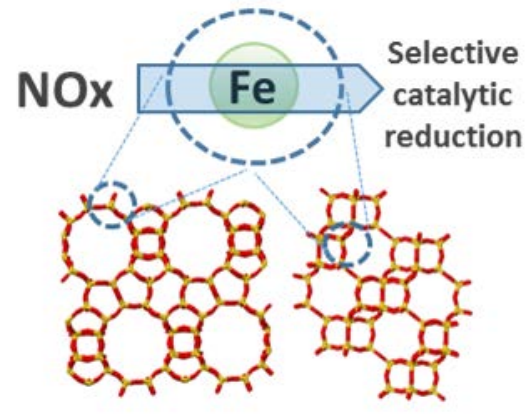

Fe-Beta Fe-CHA
Nuria Martín, Peter N. R. Vennestrøm, Joakim R. Thøgersen, Manuel Moliner, * Avelino Corma*

Page No. - Page No.

Fe-containing zeolites for $\mathrm{NH}_{3}-\mathrm{SCR}$ of NOx: effect of structure, synthesis procedure and chemical composition on catalytic performance and stability 